\title{
Public Health Surveillance in Pilot Drinking Water Contamination Warning Systems
}

\author{
Chrissy Dangel*1, Steven C. Allgeier ${ }^{1}$, Darcy Gibbons ${ }^{2}$ and Adam Haas ${ }^{2}$ \\ 'US EPA, Cincinnati, OH, USA; ${ }^{2} \mathrm{CSC}$ Science \& Engineering, Alexandria, VA, USA
}

\section{Objective}

This paper describes the lessons learned from operation and maintenance of the public health surveillance (PHS) component of five pilot city drinking water contamination warning systems (CWS) including: Cincinnati, New York, San Francisco, Philadelphia, and Dallas.

\section{Introduction}

The U.S. Environmental Protection Agency (EPA) designed a program to pilot multi-component contamination warning systems (CWSs), known as the "Water Security initiative (WSi)." The Cincinnati pilot has been fully operational since January 2008, and an additional four pilot utilities will have their own, custom CWSs by the end of 2012. A workshop amongst the pilot cities was conducted in May 2012 to discuss lessons learned from the design, implementation, operation, maintenance, and evaluation of each city's PHS component.

\section{Methods}

When evaluating potential surveillance tools to integrate into a drinking water contamination warning system, it is important to consider design decisions, dual use applications/considerations, and the unique capabilities of each tool. The pilot cities integrated unique surveillance tools, which included a combination of automated event detection tools and communication and coordination procedures into their respective PHS components. The five pilots performed a thorough, technical evaluation of each component of their CWS, including PHS.

\section{Results}

Four key lessons learned were identified from implementation of the PHS component in the five pilot cities. First, improved communication and coordination between public health and water utilities was emphasized as an essential goal even if it were not feasible to implement automated surveillance systems. The WSi pilot project has helped to strengthen this communication pathway through the process of collaborating to develop the component, and through the need to investigate PHS alerts.

Second, the approximate location of specific cases associated with PHS alerts was found to be an essential feature that allowed a crosscomparison to water pressure zones when attempting to locate the source of possible contamination. More specific location data (e.g., latitude and longitude) leads to a more efficient investigation, however, just narrowing the case location down to a specific hydraulic region within the water distribution system is extremely useful.
Third, the ability to quickly visualize spatial distribution of cases via a visual interface was reported to be valuable to investigators during alert investigations. Most pilots implemented a CWS dashboard, in the form of a central graphical display, which presents the alerts and was used by the water utility and public health to obtain an understanding of geospatial relationships between cases, alerts and water pressure zones.

Finally, public health and water utility representatives from several of the WSi pilots acknowledged that their automated surveillance tools currently have limited capabilities for detection of chemical contaminants (which may result in a sudden onset of symptoms), with the main deficiency being the timeliness of the alerts relative to the window of opportunity to respond in a meaningful and effective manner. While they currently focus on detection of traditional waterborne diseases, these tools could potentially be adapted to also detect chemical contaminants.

\section{Conclusions}

The results of the pilots have demonstrated that it is important to construct and formalize standard operating procedures, so that public health personnel and water utilities have a standard communication protocol. As a basic step to a PHS component, it is important to establish a relationship between utilities and public health. In addition to the efforts of the WSi pilots, research is currently being conducted by the U.S. EPA to analyze health seeking behavior of symptomatic individuals, because all PHS tools rely on data generated from behavior pursued by the affected population during a public health incident. Results from analysis of both emergency department data and poison control center follow-up phone data are currently underway.

\section{Keywords}

Evaluation; Public Health Surveillance; Lessons Learned; Contamination Warning System; Drinking Water

\section{References}

[1] US EPA. 2008. Water Security Initiative: Cincinnati Pilot Post-Implementation System Status Report. EPA 817-R-08-004.

*Chrissy Dangel

E-mail: dangel.chrissy@epa.gov 